\title{
DETECTION OF A NEARBY HALO DEBRIS STREAM IN THE WISE AND 2MASS SURVEYS
}

\author{
Carl J. Grillmair ${ }^{1}$, Roc Cutri ${ }^{2}$, Frank J. Masci ${ }^{2}$, Tim Conrow $^{2}$, Branimir Sesar ${ }^{3}$, \\ Peter R. M. EISENHARdT ${ }^{4}$, AND EdWARD L. WRight ${ }^{5}$ \\ ${ }^{1}$ Spitzer Science Center, 1200 E. California Blvd., Pasadena, CA 91125, USA; carl@ipac.caltech.edu \\ ${ }^{2}$ Infrared Processing and Analysis Center, California Institute of Technology, Pasadena, CA 91125, USA; \\ roc@ipac.caltech.edu,fmasci@ipac.caltech.edu,tim@ipac.caltech.edu \\ ${ }^{3}$ Division of Physics, Mathematics, and Astronomy, California Institute of Technology, Pasadena, CA 91125, USA; bsesar@astro.caltech.edu \\ ${ }^{4}$ Jet Propulsion Laboratory, California Institute of Technology, MS 169-327, 4800 Oak Grove Drive, Pasadena, CA 91109, USA; peter.r.eisenhardt@jpl.nasa.gov \\ 5 Department of Physics, University of California, Los Angeles, CA 90095, USA; wright@astro.ucla.edu \\ Received 2013 January 12; accepted 2013 April 1; published 2013 May 10
}

\begin{abstract}
Combining the Wide-Field Infrared Survey Explorer All-Sky Release with the Two Micron All Sky Survey Point Source Catalog, we detect a nearby, moderately metal-poor stellar debris stream spanning $24^{\circ}$ across the southern sky. The stream, which we designate Alpheus, is at an estimated distance of $\sim 1.9 \mathrm{kpc}$. Its position, orientation, width, estimated metallicity, and, to some extent, its distance, are in approximate agreement with what one might expect of the leading tidal tail of the southern globular cluster NGC 288.
\end{abstract}

Key words: Galaxy: halo - Galaxy: structure - globular clusters: general - globular clusters: individual (NGC 288)

Online-only material: color figure

\section{INTRODUCTION}

Wide-field photometric surveys have enabled the identification of at least 15 stellar debris streams in the Galactic halo (Grillmair 2010; Bonaca et al. 2012; Martin et al. 2013). Roughly the same number of cold substructures have been found in velocity space (Helmi et al. 1999; Smith et al. 2009; Schlaufman et al. 2009; Williams et al. 2011). In addition to helping us reconstruct the accretion history of the Galaxy, they can also serve as sensitive probes of the Galactic potential (Law et al. 2009; Koposov et al. 2010). Globular cluster streams are particularly interesting in this regard as they are the coldest stellar substructures yet discovered (Combes et al. 1999; Odenkirchen et al. 2009; Willett et al. 2009). A large sample of such streams will ultimately enable us to map the distribution of Galactic dark matter with much greater spatial resolution than has been possible to date. Detailed analyses of these streams may also enable us to better characterize the dark matter subhalos believed to populate the Galactic halo (Yoon et al. 2010; Carlberg 2009; Carlberg et al. 2012).

In this Letter we examine the Two Micron All Sky Survey (2MASS) Point Source Catalog (Skrutskie et al. 2006) and the Wide-Field Infrared Survey Explorer (WISE) All-Sky Release (Wright et al. 2010) for evidence of nearby streams in regions of the sky as yet unexplored in other surveys. We briefly describe our analysis in Section 2. We characterize the stream in Section 3, and we discuss evidence that the stream may be associated with the globular cluster NGC 288 in Section 3.1. We make concluding remarks in Section 4.

\section{DATA ANALYSIS}

Data comprising WISE bands $W 1(3.4 \mu \mathrm{m})$ and $W 2(4.6 \mu \mathrm{m})$, along with matching 2MASS $J(1.25 \mu \mathrm{m})$ photometry, were extracted from the WISE All-Sky Release and analyzed using the matched-filter technique described by Rockosi et al. (2002) and Grillmair (2009). Matched-filtering has been used to detect halo streams to very low surface densities in Sloan Digital Sky Survey (SDSS) data (Grillmair \& Johnson 2006; Grillmair \& Dionatos 2006a, 2006b; Grillmair 2009, 2011; Bonaca et al. 2012). While 2MASS data have been used to map the Sagittarius stream using M giants (Majewski et al. 2003), both WISE and 2MASS data are more limited in this application for main-sequence stars due to their much fainter magnitudes. SDSS experience has shown that matched-filter techniques over large areas work well only if the main-sequence turnoff of a population is detectable. Moreover, while WISE goes somewhat deeper than 2MASS $J$, the $W 1-W 2$ colors of stars are very nearly degenerate compared with the photometric uncertainties. Combining WISE bands $W 1$ and $W 2$ with 2MASS $J$ improves the situation, providing both color discrimination and greater depth than is possible with 2MASS photometry alone. With a limiting magnitude of $J \approx 16.5$, the WISE and 2MASS data can probe main-sequence populations out to a distance of $\approx 4 \mathrm{kpc}$.

An additional issue with the WISE All-Sky data is the presence of significant, magnitude-dependent, photometric biases at faint magnitudes resulting from an overestimate of background levels. ${ }^{6}$ Since these biases can exceed $30 \%$ at the faintest magnitudes, this has serious consequences for filtering sources based on color. Aperture magnitudes are less affected by this bias but they are much noisier due to aperture contamination. We consequently measured this bias in $W 1$ and $W 2$ by comparing profile-fitting measurements with aperture photometry using several million well-fit, isolated stars in the region $0^{\circ}<$ R.A. $<$ $45^{\circ},-60^{\circ}<$ decl. $<-30^{\circ}$. We generated a look-up table of photometric bias versus magnitude at half-magnitude intervals and interpolated this table to compute appropriate corrections for profile-fitted photometry.

We constructed matched filters based on the Padova database of theoretical stellar isochrones (Marigo et al. 2008; Girardi et al. 2010). All stars with $0<J<16.5$ were used, and we dereddened both the WISE and 2MASS photometry as a function of position on the sky using the DIRBE/IRAS dust maps of Schlegel et al. (1998), corrected using the prescription of Schlafly \& Finkbeiner (2011). We use only stars with CC flags of 0 , photometric quality flags of "A," "B," or "C," $\chi^{2}<3.0$, and

\footnotetext{
6 http://wise2.ipac.caltech.edu/docs/release/allsky/expsup/sec6_3c.html
} 


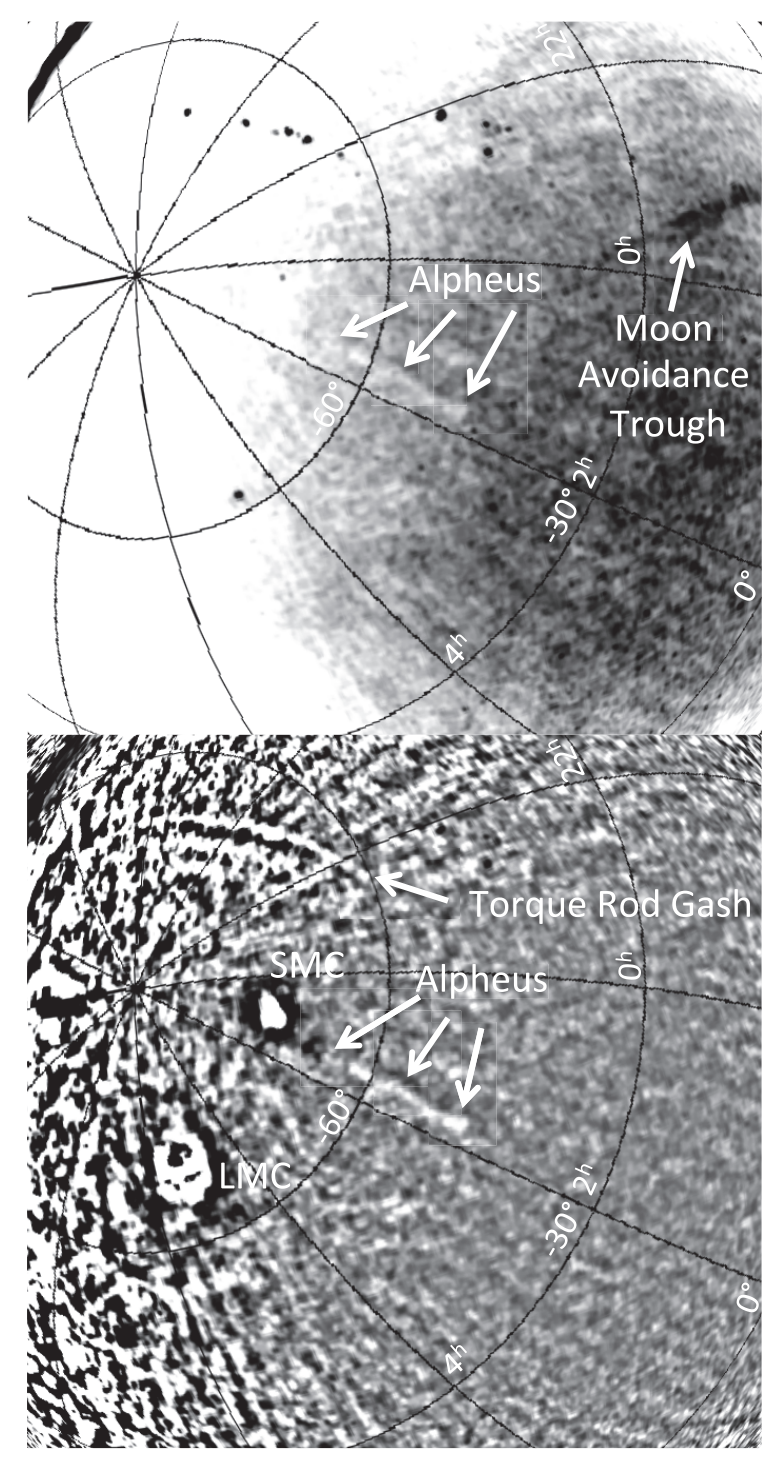

Figure 1. High contrast, filtered surface density map of WISE/2MASS stars in the south Galactic cap. The upper panel shows the map with a log stretch, smoothed with a Gaussian kernel of width 0.4 . The lighter areas indicate higher surface densities. The map is the result of a filter based on a Padova isochrone with $[\mathrm{Fe} / \mathrm{H}]=-1.0$, an age of $12 \mathrm{Gyr}$, and shifted to a distance of $1.9 \mathrm{kpc}$. In the lower panel, the map has been background-subtracted using a window-smoothed version of itself (unsharp-masking, e.g., Lee et al. 2009) to remove power at low frequencies, reduce the dynamic range, and make various features and landmarks more apparent (e.g., the LMC and SMC). The map is smoothed as above and is shown with a linear stretch. WISE-specific features resulting from moon-avoidance maneuvers and momentum dumps (repeated firing of the spacecraft torque rods at nearly the same ecliptic latitude) are indicated. The curved structure between the LMC and SMC is part of a ring-like feature surrounded the south ecliptic pole and is an artifact of image "decimation" in the WISE photometry pipeline, required in high-coverage areas due to computational limitations.

the ext flag $=0$, to select on uncontaminated, unresolved objects with signal-to-noise ratio $(\mathrm{S} / \mathrm{N})>5$. For the present search, the color-magnitude distribution of field stars was sampled using $\sim 1.2 \times 10^{7}$ stars in a broad region extending from the Small Magellanic Cloud (SMC) to the south Galactic pole. The filters were simultaneously applied to the entire WISE and 2MASS catalogs, and the resulting weights were combined to yield all-sky, filtered surface density maps.

Figure 1 shows the filtered star count distribution in the region of the south Galactic pole using a filter whose basis is an isochrone with $Z=0.0025$, an age of $12 \mathrm{Gyr}$, and is shifted so as to optimally sample populations at a distance of $1.9 \mathrm{kpc}$. This distribution was constructed by combining the weights of stars in 0.2 bins based on their distances from the $J-W 1$ and $J-W 2$ main sequence and giant branch color-magnitude loci.

\section{DISCUSSION}

Apparent in Figure 1 is a long, narrow feature spanning some $24^{\circ}$ across the southern sky. The orientation of the feature does not precisely align with lines of either constant R.A. or decl., or constant ecliptic longitude or latitude, indicating that the feature is not an artifact of sampling or data quality discontinuities in either of the two surveys. The feature shows no correlation with reddening maps of the area (where $E(B-V)$ is typically $\sim 0.02$ ), nor does it correlate with the 2 MASS j_msnr10 values, ${ }^{7}$ which estimate the depth of the 2MASS survey as a function of sky position. The feature is also localized in color-magnitude space, which would not be the case if the feature were due simply to variations in completeness. We conclude that the feature is not an artifact, but a bona fide stellar debris stream. Other curvilinear features are evident at various locations across the sky, but at much lower S/Ns.

The visible stream runs through Phoenix, Eridanus, Hydrus, Tucana, and becomes lost at its southern end near the SMC. Given the rather blue filters we are using and the magnitude limits of the WISE and 2MASS surveys, we regard it as extremely unlikely that the new stream could be associated with the SMC. Moreover, the orientation of the stream is nearly perpendicular to the SMC's orbital path (Piatek et al. 2008). We designate the new stream Alpheus, after the river in the Illiad. The northern end of the stream appears to end fairly abruptly at (R.A., decl.) $=(27.7,-45.0)$. Over the interval $-69<\delta<-45$, the path of the stream is well fit in celestial coordinates (to within $1^{\circ}$ ) by a polynomial of the form:

$$
\alpha=32.116-0.00256 \times \delta-0.00225 \times \delta^{2} .
$$

Color-magnitude Hess diagrams (CMDs) for Alpheus are shown in Figure 2. These distributions were determined using Equation (1) to select point sources (henceforth "stars") within $1^{\circ}$ of the centerline of the stream. Similar regions, $4^{\circ}$ wide on either side of the stream and laterally offset by $5^{\circ}$, were used to sample the field star population. Scaling the latter to the former by area, the CMDs in Figure 2 are subtractions of the two. While not strong, a turn-off and upper main sequence are clearly visible. For $J-W 1$, the sequence is matched reasonably well using a Padova isochrone having $[\mathrm{Fe} / \mathrm{H}]=-1.0$, though the uncertainty is quite large because the isochrones for different metallicities are rather closely spaced at these wavelengths. The $J-W 2$ CMD is less convincing, suggesting a somewhat bluer sequence. Based on the stronger $J-W 1$ sequence, Figure 2 suggests an uncertainty on the order of at least 0.5 dex. Deeper follow-up surveys in visual bands and spectroscopy of individual stars will be required to better constrain the metallicity of the stream.

A number of curvilinear features are evident in Figure 1, and one might ask how Alpheus compares in terms of significance. We can make use of the "T-statistic" of Grillmair (2009), which measures the median contrast along its length between a putative stream and the surrounding field. The T-statistic for Alpheus, comparing with the field extending the length of the stream and $30^{\circ}$ to the north and $13^{\circ}$ to the south in ecliptic coordinates, is

\footnotetext{
http://www.ipac.caltech.edu/2mass/releases/allsky/doc/sec6_2.html
} 


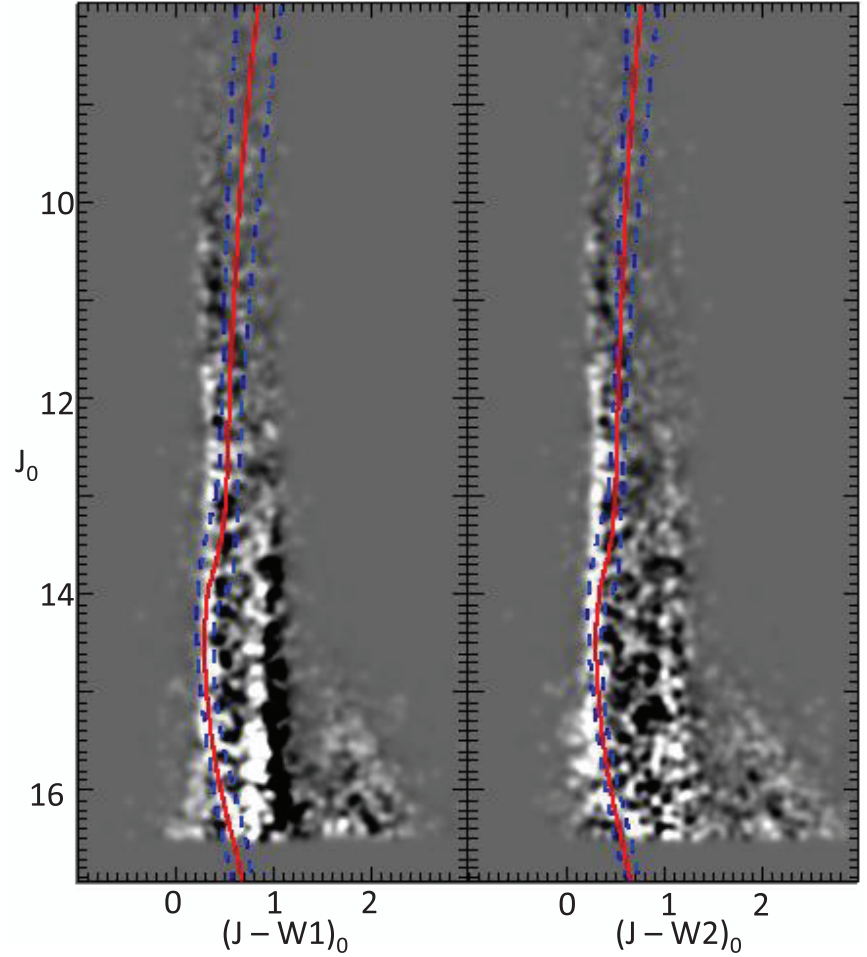

Figure 2. Hess diagrams of point sources lying within $1^{\circ}$ of the centerline of Alpheus. The solid line shows a Padova isochrone with $[\mathrm{Fe} / \mathrm{H}]=-1.0$, age $12 \mathrm{Gyr}$, and shifted to a distance of $1.9 \mathrm{kpc}$. The dashed lines show similar isochrones for $[\mathrm{Fe} / \mathrm{H}]=-2.4$ and solar metallicity. The lighter areas indicate higher surface densities.

(A color version of this figure is available in the online journal.)

shown in Figure 3. The stream is detected at the $19 \sigma$ level, and the lateral FWHM of the stream is 3.2 . At a distance of $1.9 \mathrm{kpc}$ (see below) this corresponds to a spatial extent of $\approx 100 \mathrm{pc}$. This is similar to the widths of other streams that are known or believed to have been generated by globular clusters (Odenkirchen et al. 2003; Grillmair \& Johnson 2006; Grillmair \& Dionatos 2006b; Grillmair 2009, 2011), suggesting that the progenitor of Alpheus was itself a globular cluster. The next most convincing overdensity is detected at $<10 \sigma$, and we will consider this and other features in a forthcoming paper.
Following Grillmair \& Dionatos (2006b), we shift our filter brightward and faintward to estimate the stream's distance. Combining the results for $J-W 1$ and $J-W 2$, we find that the $\mathrm{S} / \mathrm{N}$ of the northern half of the stream peaks at a distance modulus of $11.5 \pm 0.4 \mathrm{mag}$, while the southern half of the stream peaks at $11.0 \pm 0.5 \mathrm{mag}$. The uncertainties are quite large due to a combination of the small number of stars, large photometric errors at the faint limit, and the relatively narrow range of stellar colors at these wavelengths. This would put the northern half of the stream at a mean Sun-centric distance of $2.0 \pm 0.7 \mathrm{kpc}$, while the southern half is at $1.6 \pm 0.8 \mathrm{kpc}$. We note that a simple ratio of filtered stream to background signal peaks at $2.3 \mathrm{kpc}$ in the southern half and $3.9 \mathrm{kpc}$ in the northern half. However, the stream is not nearly as apparent or continuous using such a filter, nor does such a filter match the CMD in Figure 2.

Summing the color-selected, background-subtracted, but otherwise unweighted star counts over a width of 3.2 we find the number of stars in the stream to $W 1 \approx 16.5$ to be $310 \pm 90$. The quoted uncertainty is largely due to the shot noise contributed by the several thousand field stars with similar colors lying along our line of sight to the stream. The average surface density is $4 \pm 1$ stars deg $^{-2}$, with peaks of $\sim 10$ stars deg $^{-2}$.

\subsection{On the Possible Association of Alpheus with the Globular Cluster NGC 288}

Based on its relatively low concentration and fairly eccentric, retrograde orbit, NGC 288 has a relatively high predicted destruction rate (Gnedin \& Ostriker 1997), and both Grillmair et al. (1995) and Leon et al. (2000) found a significant population of extratidal stars in the vicinity of the cluster. Figure 4 shows projections of three possible orbits of the cluster, integrated using the Galactic model of Allen \& Santillan (1991). All integrations use a cluster distance of $8.3 \mathrm{kpc}$ and a radial velocity of $-47 \mathrm{~km} \mathrm{~s}^{-1}$ (Harris 1996). The three different orbits are based on proper motions of $\mu_{\alpha} \cos \delta=4.68 \pm 0.2 \mathrm{mas} \mathrm{yr}^{-1}$, $\mu_{\delta}=-5.25 \pm 0.2$ mas yr $^{-1}$ (Guo 1995) ("a"), $\mu_{\alpha} \cos \delta=$ $4.64 \pm 0.4 \mathrm{mas} \mathrm{yr}^{-1}, \mu_{\delta}=-6.00 \pm 0.4 \mathrm{mas} \mathrm{yr}^{-1}$ (Dinescu et al. 1997) ("b"), and $\mu_{\alpha} \cos \delta=3.8 \pm 0.3 \mathrm{mas} \mathrm{yr}^{-1}, \mu_{\delta}=$ $-8.1 \pm 0.3$ mas yr $^{-1}$ (Platais et al. 1998) ("c").

Two of the orbit integrations ("a" and "b") lie nearly parallel to the new stream, offset to the east by $\sim 2^{\circ}-3^{\circ}$. Orbit "c" is also nearly parallel to the stream, but is offset $\sim 7^{\circ}$ to the west. Tracing around the integrated orbits, the visible portion of the

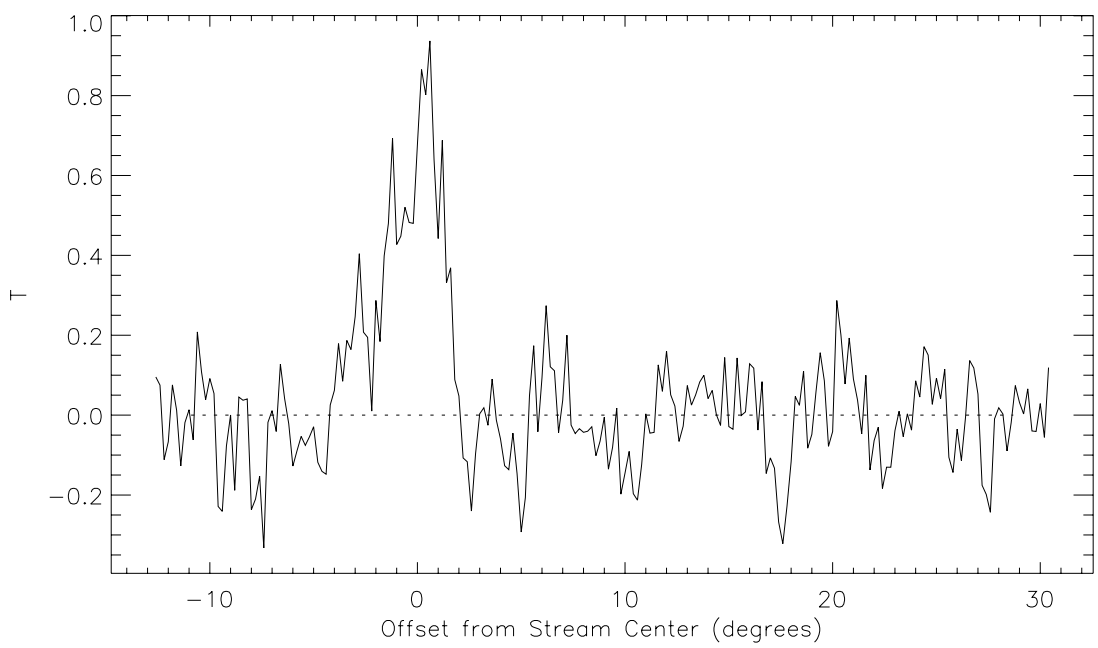

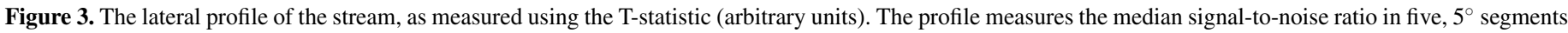
of Alpheus, where the noise is measured in fields extending $13^{\circ}$ and $30^{\circ}$ to the south and north, respectively. 


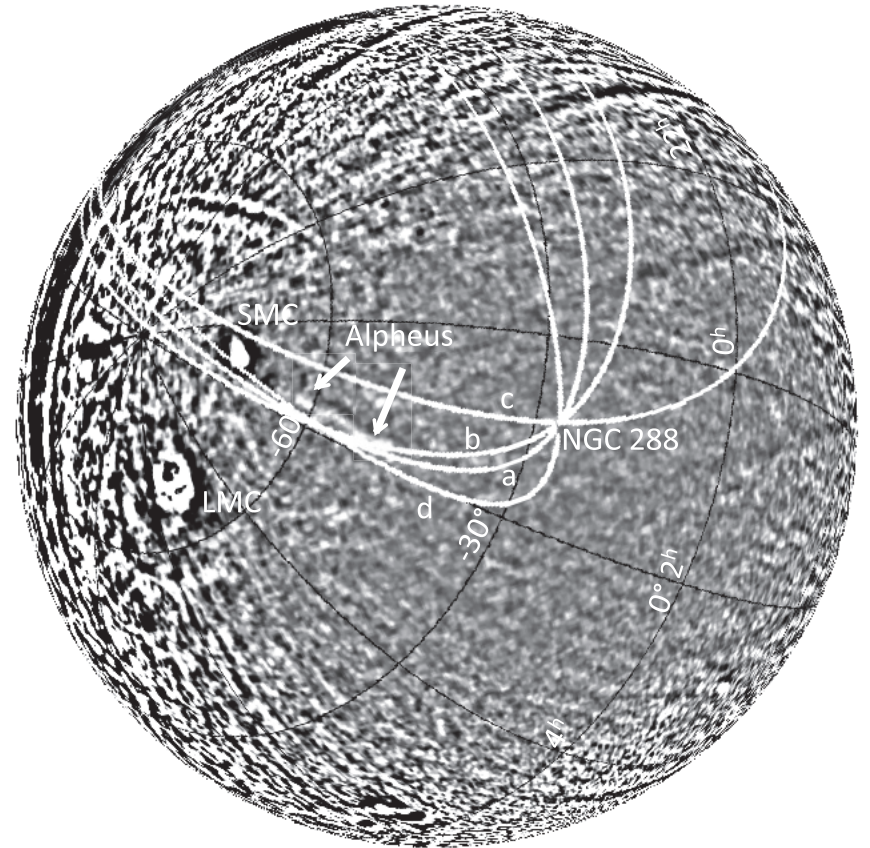

Figure 4. Projections of the orbit of NGC 288 using three different proper motions measurements (see text). Orbit " $d$ " was generated by both forcing the orbit to pass through NGC 288 and to pass 2.5 to the east along the length of Alpheus.

stream begins at $\sim 4 \mathrm{kpc}$ from the cluster and ends at $\sim 6.3 \mathrm{kpc}$. Over the $24^{\circ}$ extent of the stream, the leading arm of orbit "a" predicts a heliocentric distance ranging from $3.8 \mathrm{kpc}$ at the southern end to $5.1 \mathrm{kpc}$ at the northern end. Similarly, orbit "b" ranges from $5 \mathrm{kpc}$ to $6.2 \mathrm{kpc}$, and orbit "c" ranges from $7.2 \mathrm{kpc}$ to $7.5 \mathrm{kpc}$. The near coincidences in the position, orientation, and distance gradient of the stream, particularly with orbit "a," suggest a possible association with NGC 288.

Given the retrograde motion of NGC 288, the stream would constitute the leading arm of the NGC 288's tidal tails. We would expect these stars to have dropped inward toward the Galactic center before being carried forward along their new orbits. That the stream lies to the inside of putative orbits "a" and " $b$ " is consistent with this picture. An offset of 2.5 at a distance of $1.9 \mathrm{kpc}$ corresponds to $\approx 80 \mathrm{pc}$ in projection. Though this offset is somewhat larger than the current tidal radius of NGC 288 ( 31 pc; Harris 1996), we expect escaped stars to have residual velocities that will carry them somewhat farther from the cluster. Indeed, Grillmair et al. (1995) and Leon et al. (2000) found extratidal stars extending to at least $150 \mathrm{pc}$ in the immediate vicinity of NGC 288.

Our metallicity estimate for stars in the stream is also consistent with a measured value of $[\mathrm{Fe} / \mathrm{H}]=-1.24(\mathrm{Z} \approx$ 0.0015 ) for NGC 288 (Harris 1996). As noted earlier, while the stream appears strongest using isochrones with $Z=0.0025$ $(\mathrm{Fe} / \mathrm{H} \approx-1.0)$, due to the small number of stars and the narrow range of colors at these wavelengths, the stream is only marginally less apparent $(\mathrm{S} / \mathrm{N} \sim 17.5)$ using an isochrone with $Z=0.0015$. 2MASS and WISE colors are not very sensitive to metallicity, and given the additional uncertainty contributed by our substantial corrections to the WISE profile-fit photometry, we consider the agreement to be quite good.

If we adopt a proper motion for NGC 288 of $\mu_{\alpha} \cos \delta=$ 4.75 mas yr$^{-1}, \mu_{\delta}=-4.60$ mas yr$^{-1}$, we arrive at orbit "d" in Figure 4. This value of the proper motion was arrived at by both forcing the orbit to pass through NGC 288, and to pass 2.5 to the east along the length of Alpheus. The heliocentric distance of this orbit in the range $-65^{\circ}<\delta<-45^{\circ}$ is reduced to between 2.3 and $3.1 \mathrm{kpc}$ which, to within the uncertainties, is in agreement with our distance estimate for the stream. The adopted value of $\mu_{\alpha} \cos \delta$ is in good agreement with the measured values, while the value of $\mu_{\delta}$ is $1.2 \sigma$ from the mean of the three measurements. Combining the measurement uncertainties with inaccuracies in our adopted model for the Galactic potential, we consider the association of the stream with NGC 288 plausible, if not proven. Validation of our hypothesis will require radial velocity measurements for a significant number of stream stars.

That the stream does not visibly connect with NGC 288 in Figure 4 may simply be due to the limiting magnitude of the WISE and 2MASS surveys. At the northernmost point of the visible stream, the NGC 288 orbit model predicts that the heliocentric distances rapidly increase to $8.3 \mathrm{kpc}$ at the position of NGC 288 . As we use our filters to probe beyond $3-5 \mathrm{kpc}$, the turn-off and subgiant branch fall below our limiting magnitude and we are forced to rely wholly on the red giant branch (RGB) for our signal. However, the RGB is much more sparsely populated and more highly contaminated by foreground stars than is the turn-off region. We detect NGC 288 only by virtue of the relatively large number of RGB stars in the cluster itself.

It is also possible that the stream has been partially disrupted by a dark matter subhalo or other perturber of significant mass and/or favorable trajectory. However, examinations of the Pal 5 and GD-1 streams (Carlberg et al. 2012; Carlberg \& Grillmair 2013) have not identified gaps anywhere near $4 \mathrm{kpc}$ in length. We consequently consider this an unlikely possibility, but one that will need to be tested with deeper observations.

\section{CONCLUSION}

Using the 2MASS and WISE All-Sky Catalogs, we identify a new, $24^{\circ}$ long, moderately metal-poor stellar debris stream in the southern hemisphere which we designate Alpheus. At a distance of $\approx 1.9 \mathrm{kpc}$, the stream is much closer than other spatially mapped streams that have been found to date.

Based on near coincidences in position, orientation, distance, distance gradient, and metallicity, we suggest that the stream may be the leading tidal arm of the globular cluster NGC 288. Testing this hypothesis will require both deeper imaging in the visible bands and spectroscopy. Deeper imaging by the SkyMapper survey (Keller et al. 2007) should enable us to reach well down the main sequence to better characterize the stream and extend its path across the sky. Based on existing proper motion measurements for NGC 288 as well as our attempt to estimate an orbit that would connect Alpheus with the cluster, we would predict radial velocities in the range $-66<v_{r}<-36 \mathrm{~km} \mathrm{~s}^{-1}$ at the northern end of the stream, and $23<v_{r}<36 \mathrm{~km} \mathrm{~s}^{-1}$ at the southern end. If Alpheus is indeed associated with NGC 288, these measurements will also enable more comprehensive modeling of the orbit of NGC 288 and the shape of the Galactic potential toward the south Galactic pole.

We gratefully acknowledge several probing questions by an anonymous referee which helped us to improve and clarify the manuscript. We also thank S. van den Bergh for suggesting a new source of stream designations. This paper is based upon work supported in part by NASA under award No. NNX12AI57G. 
This publication makes use of data products from the WideField Infrared Survey Explorer, which is a joint project of the University of California, Los Angeles, and the Jet Propulsion Laboratory/California Institute of Technology, funded by the National Aeronautics and Space Administration. It also makes use of data products from the Two Micron All Sky Survey, which is a joint project of the University of Massachusetts and the Infrared Processing and Analysis Center, funded by the National Aeronautics and Space Administration and the National Science Foundation.

Facility: WISE

\section{REFERENCES}

Allen, C., \& Santillan, A. 1991, RMxAA, 22, 255

Bonaca, A., Geha, M., \& Kallivayalil, N. 2012, ApJ, 760, 6

Carlberg, R. G. 2009, ApJ, 705, 223

Carlberg, R. G., \& Grillmair, C. J. 2013, ApJ, 768, 171

Carlberg, R. G., Grillmair, C. J., \& Hetherington, N. 2012, ApJ, 760, 75

Combes, F., Leon, S., \& Meylan, G. 1999, A\&A, 352, 149

Dinescu, D. I., Girard, T. M., van Altena, W. F., Mendez, R. A., \& Lopez, C. E. 1997, AJ, 114, 1014

Girardi, L., Williams, B. F., Gilbert, K. M., et al. 2010, ApJ, 724, 1030

Gnedin, O. Y., \& Ostriker, J. P. 1997, ApJ, 474, 223

Grillmair, C. J. 2009, ApJ, 693, 1118

Grillmair, C. J. 2010, in Galaxies and Their Masks, ed. D. Block, K. C. Freeman, \& I. Puerari (New York: Springer), 247

Grillmair, C. J. 2011, ApJ, 738, 98

Grillmair, C. J., \& Dionatos, O. 2006a, ApJL, 641, L37
Grillmair, C. J., \& Dionatos, O. 2006b, ApJL, 643, L17

Grillmair, C. J., Freeman, K. C., Irwin, M., \& Quinn, P. J. 1995, AJ, 109, 2553

Grillmair, C. J., \& Johnson, R. 2006, ApJL, 639, L17

Guo, X. 1995, PhD thesis, Yale Univ.

Harris, W. E. 1996, AJ, 112, 1487

Helmi, A., White, S. D. M., de Zeeuw, P. T., \& Zhao, H. 1999, Natur, 402, 53

Keller, S. C., Schmidt, B. P., Bessell, M. S., et al. 2007, PASA, 24, 1

Koposov, S. E., Rix, H.-W., \& Hogg, D. W. 2010, ApJ, 712, 260

Law, D. R., Majewski, S. R., \& Johnston, K. V. 2009, ApJ, 703, 67

Lee, M., Stanimirović, S., Ott, J., et al. 2009, AJ, 138, 1101

Leon, S., Meylan, G., \& Combes, F. 2000, A\&A, 359, 907

Majewski, S. R., Skrutskie, M. F., Weinberg, M. D., \& Ostheimer, J. C. 2003, ApJ, 599, 1082

Marigo, P., Girardi, L., Bressan, A., et al. 2008, A\&A, 482, 883

Martin, C., Carlin, J. L., Newberg, H. J., \& Grillmair, C. J. 2013, ApJ, 765, 39

Odenkirchen, M., Grebel, E. K., Dehnen, W., et al. 2003, AJ, 126, 2385

Odenkirchen, M., Grebel, E. K., Kayser, A., Rix, H.-W., \& Dehnen, W. 2009, AJ, 137,3378

Piatek, S., Pryor, C., \& Olszewski, E. W. 2008, AJ, 135, 1024

Platais, I., Girard, T. M., Kozhurina-Platais, V., et al. 1998, AJ, 116, 2556

Rockosi, C. M., Odenkirchen, M., Grebel, E. K., et al. 2002, AJ, 124, 349

Schlafly, E. F., \& Finkbeiner, D. P. 2011, ApJ, 737, 103

Schlaufman, K. C., Rockosi, C. M., Allende Prieto, C., et al. 2009, ApJ, 703,2177

Schlegel, D. J., Finkbeiner, D. P., \& Davis, M. 1998, ApJ, 500, 525

Skrutskie, M. F., Cutri, R. M., Stiening, R., et al. 2006, AJ, 131, 1163

Smith, M. C., Evans, N. W., Belokurov, V., et al. 2009, MNRAS, 399, 1223

Willett, B. A., Newberg, H. J., Zhang, H., Yanny, B., \& Beers, T. 2009, ApJ, 697, 207

Williams, M. E. K., Steinmetz, M., Sharma, S., et al. 2011, ApJ, 728, 102

Wright, E., Eisenhardt, P. R. M., Mainzer, A. K., et al. 2010, AJ, 140, 1868

Yoon, J. H., Johnston, K. V., \& Hogg, D. W. 2011, ApJ, 741, 58 